TRANSACTIONS OF THE

AMERICAN MATHEMATICAL SOCIETY

Volume 359, Number 3, March 2007, Pages 1265-1284

S 0002-9947(06)03925-0

Article electronically published on August 24, 2006

\title{
TORIC HYPERSYMPLECTIC QUOTIENTS
}

\author{
ANDREW DANCER AND ANDREW SWANN
}

\begin{abstract}
We study the hypersymplectic spaces obtained as quotients of flat hypersymplectic space $\mathbb{R}^{4 d}$ by the action of a compact Abelian group. These $4 n$-dimensional quotients carry a multi-Hamilitonian action of an $n$ torus. The image of the hypersymplectic moment map for this torus action may be described by a configuration of solid cones in $\mathbb{R}^{3 n}$. We give precise conditions for smoothness and non-degeneracy of such quotients and show how some properties of the quotient geometry and topology are constrained by the combinatorics of the cone configurations. Examples are studied, including non-trivial structures on $\mathbb{R}^{4 n}$ and metrics on complements of hypersurfaces in compact manifolds.
\end{abstract}

\section{INTRODUCTION}

An important construction in symplectic geometry is the symplectic quotient of Marsden and Weinstein. Given a symplectic action of a Lie group $G$ on a symplectic manifold $M$, this produces, under fairly mild hypotheses, a new symplectic manifold of dimension $\operatorname{dim} M-2 \operatorname{dim} G$.

One particularly nice class of symplectic examples is that of toric varieties. Delzant [De and Guillemin [Gu], have shown that a large class of toric varieties may be produced as symplectic quotients of $\mathbb{C}^{d}$ with its flat Kähler structure by a subtorus $N$ of $\mathbb{T}^{d}$. Their work also shows that the symplectic and Kähler geometry of these $2 n$-dimensional examples is determined by concrete descriptions of the moment polytope as an intersection of closed half-spaces in $\mathbb{R}^{n}$.

In the present paper we shall investigate an analogous construction in a situation where there are multiple symplectic structures, namely for hypersymplectic manifolds. We consider the geometries arising from a hypersymplectic quotient construction for compact Abelian subgroups $N$ of $\mathbb{T}^{d}$ acting on $\mathbb{C}^{d, d}=\mathbb{C}^{d} \times \mathbb{C}^{d}$. We determine conditions for the quotients to be smooth manifolds and to admit non-degenerate geometric structures in two ways: firstly by direct considerations, and then by using the (hypersymplectic) moment map for the action of a torus on the quotient. The closed half-spaces of Delzant and Guillemin are now replaced by solid cones in $\mathbb{R}^{3 n}$, and we demonstrate how properties of the quotient may be deduced from particular descriptions of such cone configurations.

Hypersymplectic structures were defined in a paper of Hitchin $[\mathrm{H}]$ and have appeared in recent works such as $[\mathrm{Hu}, \mathrm{Ka}, \mathrm{FPPW}, \mathrm{AD}$. Hitchin's work is motivated by the geometry of the moduli space of harmonic maps into a compact Lie group;

Received by the editors September 29, 2004 and, in revised form, December 21, 2004.

2000 Mathematics Subject Classification. Primary 53C25; Secondary 53D20, 53C55, 57S15.

Key words and phrases. Hypersymplectic structure, neutral hyperkähler manifold, toric variety, moment map.

(C)2006 American Mathematical Society
Reverts to public domain 28 years from publication 1265 
the paper of Hull $[\mathrm{Hu}]$ and the references therein show the role played by these structures in string theory; Mason and Sparling encountered such structures whilst studying the non-linear Schrödinger and the Korteweg-de Vries equations MS. On a hypersymplectic manifold one has an indefinite Kähler metric $g$ of signature $(2 n, 2 n)$, together with a covariant constant endomorphism $S$ of the tangent bundle, such that $S^{2}$ equals the identity, $S$ anti-commutes with the complex structure $I$, and $g(S X, S Y)=-g(X, Y)$. Now $I, S$ and $T=I S$ generate an action of the Lie algebra $\mathfrak{s l}(2, \mathbb{R})$ on each tangent space. Moreover, $I, S, T$, together with the metric $g$, define three symplectic forms by

$$
\begin{gathered}
\omega_{I}(X, Y)=g(X, I Y), \quad \omega_{S}(X, Y)=g(X, S Y), \\
\omega_{T}(X, Y)=g(X, T Y),
\end{gathered}
$$

hence the name "hypersymplectic". Every hypersymplectic manifold is neutral Calabi-Yau, that is, Ricci-flat Kähler with signature $(2 n, 2 n)$. Hypersymplectic manifolds are split-quaternion analogues of hyperkähler manifolds and are sometimes referred to as "neutral hyperkähler manifolds".

Hitchin described a quotient construction for hypersymplectic manifolds in $[\mathrm{H}]$, analogous to the hyperkähler quotient of [HKLR]. If $G$ acts preserving a hypersymplectic structure, then under mild conditions, we have, for each $X \in \mathfrak{g}$, a moment map $\mu^{X}$ taking values in $\mathbb{R}^{3}$. This map satisfies the defining equation

$$
d \mu^{X}(Y)=\left(\omega_{I}(X, Y), \omega_{S}(X, Y), \omega_{T}(X, Y)\right),
$$

where we identify $X$ with the vector field it induces via the group action. Of course, (1.1) only gives $\mu^{X}$ up to an additive constant; these constants are partially restricted by the additional assumption that the maps $\mu^{X}$ combine to define a $G$-equivariant map $\mu$ taking values in $\mathfrak{g}^{*} \otimes \mathbb{R}^{3}$. If $G$ is Abelian, then any choice of $\mu^{X}$ gives an equivariant map. The hypersymplectic quotient is now defined to be $\mu^{-1}(0) / G$. When $\mu$ has maximal rank and the action of $G$ is free, the quotient has dimension $4 \operatorname{dim} G$ less than the original hypersymplectic manifold. It inherits closed two-forms from $\omega_{I}, \omega_{S}$ and $\omega_{T}$, and one expects these to define a hypersymplectic structure; however degeneracies may occur on a certain locus in the quotient.

In this paper we shall concentrate on hypersymplectic quotients of flat space $\mathbb{C}^{d, d}$ by compact Abelian groups, although we prove some general results controlling smoothness and non-degeneracy of arbitrary hypersymplectic quotients. In some ways, the picture is intermediate between that of Kähler quotients and of hyperkähler quotients as studied in $\mathrm{BD}$. In the hyperkähler case the quotients are necessarily non-compact, whereas in the Kähler case many quotients are compact. For the hypersymplectic situation, we show how to produce non-compact non-singular structures on $\mathbb{R}^{4 n}$ that are not flat. It is also easy to produce compact quotient sets, and with a little more work smooth examples may be found, but on the other hand these always have singularities of the hypersymplectic structure. We show that these compact quotients produce non-degenerate structures on hypersurface complements in real analytic subvarieties of compact toric varieties. All the quotients we produce carry a natural involution. We discuss in detail some particular examples, such as the hypersymplectic analogues of the Calabi and Gibbons-Hawking multi-instanton spaces, and all examples obtainable as quotients of $\mathbb{C}^{2,2}$ by a one-dimensional group. 


\section{THE FLAT HYPERSYMPLECTIC STRUCTURE}

Our examples will be hypersymplectic quotients of the following flat hypersymplectic structure. Let $\mathbb{C}^{d, d}$ be $\mathbb{C}^{d} \times \mathbb{C}^{d}$ with the complex structure

$$
I(z, w)=(z \mathbf{i},-w \mathbf{i}),
$$

where $\mathbf{i}=\sqrt{-1}$, and with the indefinite Kähler metric

$$
g=\operatorname{Re}\left(\sum_{k=1}^{d} d z_{k} d \bar{z}_{k}-d w_{k} d \bar{w}_{k}\right) .
$$

Note that $I$ is not the standard complex structure $I_{0}$ on this space, which is instead defined by $I_{0}:(z, w) \mapsto(z \mathbf{i}, w \mathbf{i})$. To distinguish $I$ from $I_{0}$, we shall refer to this space with complex structure $I_{0}$ as $\mathbb{C}^{2 d}$.

If we define $S(z, w)=(w, z)$, then

$$
\begin{gathered}
I S(z, w)=I(w, z)=(w \mathbf{i},-z \mathbf{i}), \\
S I(z, w)=S(z \mathbf{i},-w \mathbf{i})=(-w \mathbf{i}, z \mathbf{i}),
\end{gathered}
$$

so $I S=-S I$. We define $T=I S$, so that

$$
I^{2}=-1, \quad S^{2}=T^{2}=1, \quad I S=T=-S I .
$$

We have the following symplectic forms:

$$
\begin{aligned}
& \omega_{I}=\frac{1}{2 \mathbf{i}} \sum_{k=1}^{d}\left(d z_{k} \wedge d \bar{z}_{k}+d w_{k} \wedge d \bar{w}_{k}\right), \\
& \omega_{S}=\frac{1}{2} \sum_{k=1}^{d}\left(d z_{k} \wedge d \bar{w}_{k}-d w_{k} \wedge d \bar{z}_{k}\right), \\
& \omega_{T}=\frac{1}{2 \mathbf{i}} \sum_{k=1}^{d}\left(d z_{k} \wedge d \bar{w}_{k}+d w_{k} \wedge d \bar{z}_{k}\right) .
\end{aligned}
$$

Note that $\omega_{S}+\mathbf{i} \omega_{T}=\sum_{k=1}^{d} d z_{k} \wedge d \bar{w}_{k}$, which is a holomorphic $(2,0)$-form with respect to $I$, but is of type $(1,1)$ for $I_{0}$.

\section{Moment maps}

The torus $\mathbb{T}^{d}$ acts on $\mathbb{C}^{d, d}$ by

$$
\left(z_{k}, w_{k}\right) \longmapsto\left(e^{\mathbf{i} \theta_{k}} z_{k}, e^{\mathbf{i} \theta_{k}} w_{k}\right) .
$$

This action commutes with $I$ and $S$ and hence with $T$, and preserves $g$.

The moment maps from (1.1) are

$$
\begin{gathered}
\mu_{I}:(z, w) \longmapsto \sum_{k=1}^{d} \frac{1}{2}\left(\left|z_{k}\right|^{2}+\left|w_{k}\right|^{2}\right) e_{k}+\tilde{c}_{1}, \\
\mu_{S}+\mathbf{i} \mu_{T}:(z, w) \longmapsto \sum_{k=1}^{d} \mathbf{i} z_{k} \bar{w}_{k} e_{k}+\tilde{c}_{2}+\mathbf{i} \tilde{c}_{3},
\end{gathered}
$$

where $d \mu_{I}^{X}(Y)=\omega_{I}(X, Y)$, etc., $e_{1}, \ldots, e_{d}$ are the standard basis vectors for $\mathbb{R}^{d}$, and $\tilde{c}_{1}, \tilde{c}_{2}, \tilde{c}_{3}$ are arbitrary constant vectors in $\mathbb{R}^{d}$. 
The form of this differs from the hyperkähler moment map $\mathrm{BD}$ for the $\mathbb{T}^{d}$ action on $\mathbb{H}^{d}$, in that we have $+\left|w_{k}\right|^{2}$ rather than $-\left|w_{k}\right|^{2}$ in the formula for $\mu_{I}$. We also recall, for comparison, that the Kähler moment map for the action of $\mathbb{T}^{d}$ on $\mathbb{C}^{d}$ is $\mu: z \mapsto \sum_{k=1}^{d} \frac{1}{2}\left|z_{k}\right|^{2} e_{k}+c$.

As in $\mathrm{Gu}, \mathrm{BD}$ one considers a compact Abelian subgroup $N$ of $\mathbb{T}^{d}$ with Lie algebra $\mathfrak{n}$. We shall take $\mathfrak{n}$ to be the kernel of a surjective linear map $\beta: \mathbb{R}^{d} \rightarrow \mathbb{R}^{n}$ given by

$$
\beta: e_{k} \longmapsto u_{k}
$$

with $u_{i} \in \mathbb{Z}^{n}$. In particular, $\mathbb{R}^{n}$ is spanned by $u_{1}, \ldots, u_{d}$. Then we have an exact sequence

$$
0 \longrightarrow \mathfrak{n} \stackrel{\iota}{\longrightarrow} \mathbb{R}^{d} \stackrel{\beta}{\longrightarrow} \mathbb{R}^{n} \longrightarrow 0
$$

and $N$ is defined to be the kernel of the map $\exp \beta \circ \exp ^{-1}: \mathbb{T}^{d} \rightarrow \mathbb{T}^{n}$. (The requirement that $u_{i}$ be integral exactly guarantees that this composition is well defined.) For a given $N$, the map $\beta$ is unique up to composition with an element of $\operatorname{Aut}\left(\mathbb{Z}^{n}\right)$, or in matrix terms up to multiplication by an element of $G L(n, \mathbb{Z})=$ $\left\{A \in M_{n}(\mathbb{Z}): \operatorname{det} A \neq 0\right.$ and $\left.A^{-1} \in M_{n}(\mathbb{Z})\right\}$.

We let $\langle\cdot, \cdot\rangle$ denote the standard inner product with respect to which $e_{1}, \ldots, e_{d}$ are orthonormal. For each choice of scalars $\lambda_{1}, \ldots, \lambda_{d}$, the set of vectors $\left\{u_{1}, \ldots, u_{d}\right\}$ defines a convex polyhedron in $\mathbb{R}^{n}$ by the equations

$$
\left\langle s, u_{k}\right\rangle \geqslant \lambda_{k}, \quad \text { for } k=1, \ldots, d .
$$

In general, this polyhedron may be non-compact.

There is an exact sequence dual to (3.1)

$$
0 \longrightarrow \mathbb{R}^{n *} \stackrel{\beta^{*}}{\longrightarrow} \mathbb{R}^{d^{*}} \stackrel{\iota^{*}}{\longrightarrow} \mathfrak{n}^{*} \longrightarrow 0
$$

We shall identify $\mathbb{R}^{d^{*}}$ with $\mathbb{R}^{d}$ using $\langle\cdot, \cdot\rangle$. Now $\beta^{*}$ is given by

$$
\beta^{*}(a)=\sum_{k=1}^{d}\left\langle a, u_{k}\right\rangle e_{k}
$$

and the moment map for $N$ becomes

$$
\begin{gathered}
\mu_{I}:(z, w) \longmapsto \sum_{k=1}^{d} \frac{1}{2}\left(\left|z_{k}\right|^{2}+\left|w_{k}\right|^{2}\right) \alpha_{k}+c_{1}, \\
\mu_{S}+\mathbf{i} \mu_{T}:(z, w) \longmapsto \sum_{k=1}^{d} \mathbf{i} z_{k} \bar{w}_{k} \alpha_{k}+c_{2}+\mathbf{i} c_{3},
\end{gathered}
$$

where $\alpha_{k}=\iota^{*} e_{k}$. We write $c_{j}=\sum_{k=1}^{d} \lambda_{k}^{(j)} \alpha_{k}$ for some scalars $\lambda_{k}^{(j)}$.

A point $(z, w)$ lies in $\mu_{I}^{-1}(0)$ if and only if

$$
\iota^{*}\left(\sum_{k=1}^{d}\left(\frac{1}{2}\left(\left|z_{k}\right|^{2}+\left|w_{k}\right|^{2}\right)+\lambda_{k}^{(1)}\right) e_{k}\right)=0 .
$$

However $\operatorname{ker} \iota^{*}=\operatorname{im} \beta^{*}$, so using (3.3), we see that (3.4) is equivalent to the existence of $a \in \mathbb{R}^{n}$ such that

$$
\left\langle a, u_{k}\right\rangle=\frac{1}{2}\left(\left|z_{k}\right|^{2}+\left|w_{k}\right|^{2}\right)+\lambda_{k}^{(1)}, \quad \text { for } k=1, \ldots, d .
$$


Similarly, $(z, w) \in\left(\mu_{S}+\mathbf{i} \mu_{T}\right)^{-1}(0)$ if and only if

$$
\left\langle b, u_{k}\right\rangle=\mathbf{i} z_{k} \bar{w}_{k}+\lambda_{k}^{(2)}+\mathbf{i} \lambda_{k}^{(3)}, \quad \text { for } k=1, \ldots, d,
$$

for some $b \in \mathbb{C}^{n}$.

Equations (3.5) and (3.6) give a description of the level set $\mu^{-1}(0)$. The hypersymplectic quotient $M$ of $\mathbb{C}^{d, d}$ by $N$ is defined to be

$$
M=\mu^{-1}(0) / N \text {. }
$$

This is a Hausdorff topological space; as we will see it may or may not be a smooth manifold.

\section{NON-DEgEnERACY OF THE QUOTIENT GEOMETRY}

In this section we shall consider when hypersymplectic quotients are smooth, and at which points the hypersymplectic structure on the quotient can degenerate. We will begin with the general case and then specialise to torus quotients of flat space.

Let us consider an action of a Lie group $G$ on a manifold of dimension $4 d$ preserving a hypersymplectic structure and admitting a $G$-equivariant moment map $\mu: M \rightarrow \mathfrak{g}^{*} \otimes \mathbb{R}^{3}$. Thus, for each element $X$ of $\mathfrak{g}$, the associated component $\mu^{X}$ of $\mu$ satisfies (1.1). Write $\mathcal{G}$ for the distribution on $M$ generated by tangent vectors to the group action.

Definition 4.1. The action satisfies condition (F) if $G$ acts properly and freely on $\mu^{-1}(0)$.

Definition 4.2. The action satisfies condition (S) if at each point $p \in \mu^{-1}(0)$ there is no non-zero solution to the equation

$$
\left(I X_{1}+S X_{2}+T X_{3}\right)_{p}=0
$$

with $X_{1}, X_{2}, X_{3} \in \mathfrak{g}$.

Theorem 4.3. If the G-action satisfies conditions $(\mathrm{F})$ and $(\mathrm{S})$, then the hypersymplectic quotient $\mu^{-1}(0) / G$ is smooth.

Proof. It is sufficient, by (F), to show that $\mu^{-1}(0)$ is a smooth manifold. By (1.1), the kernel ker $d \mu$ is just the orthogonal complement with respect to $g$ of the space $U$ spanned by $I \mathcal{G}, S \mathcal{G}$ and $T \mathcal{G}$.

As $g$ is non-degenerate we have

$$
\operatorname{rank} d \mu=4 d-\operatorname{dim} \operatorname{ker} d \mu=4 d-\operatorname{dim} U^{\perp}=\operatorname{dim} U .
$$

Condition (S) implies that $\operatorname{dim} U$ is $3 \operatorname{dim} \mathcal{G}$, which is $3 \operatorname{dim} G$ by $(\mathrm{F})$. We deduce that $d \mu$ has maximal rank, and the result follows.

Remark 4.4. As the moment map $\mu$ is equivariant, $\mathcal{G}$ lies in $\operatorname{ker} d \mu$ on $\mu^{-1}(0)$. This implies that $\mathcal{G}$ is orthogonal to $I \mathcal{G}, S \mathcal{G}$ and $T \mathcal{G}$. It follows that these spaces are mutually orthogonal.

In the hyperkähler case, where $g$ is positive definite, this of course means that condition (S) and the conclusion of the theorem follow automatically from the freeness of the action of $G$, as in [HKLR]. 
It is proved in $[\mathrm{H}]$ that the kernels of the symplectic forms on $\mu^{-1}(0)$ are given by

$$
\operatorname{ker} i^{*} \omega_{I}=\mathcal{G}+S\left(\mathcal{G} \cap \mathcal{G}^{\perp}\right)+T\left(\mathcal{G} \cap \mathcal{G}^{\perp}\right)
$$

and cyclically, where $i: \mu^{-1}(0) \rightarrow M$ is inclusion.

Definition 4.5. The action satisfies condition (D) if $\mathcal{G} \cap \mathcal{G}^{\perp}=\{0\}$ on $\mu^{-1}(0)$.

Theorem 4.6. If the $G$-action satisfies conditions $(\mathrm{F})$ and $(\mathrm{D})$, then the quotient $\mu^{-1}(0) / G$ inherits a smooth, non-degenerate hypersymplectic structure.

On the other hand, if the $G$-action fulfils conditions $(\mathrm{F})$ and $(\mathrm{S})$, then the smooth manifold $\mu^{-1}(0) / G$ inherits a non-degenerate hypersymplectic structure only if condition (D) is satisfied.

Proof. Hitchin's results provide a non-degenerate hypersymplectic structure provided $\mu^{-1}(0) / G$ is a smooth manifold and (D) holds: the symplectic form $\omega_{I}^{\prime}$ on the quotient is defined by the equation $\pi^{*} \omega_{I}^{\prime}=i^{*} \omega_{I}$, where $\pi$ is projection from $\mu^{-1}(0)$ to the quotient. Thus for the first part of the theorem we only need to consider condition (S) of Definition 4.2 .

Suppose $I X_{1}+S X_{2}+T X_{3}=0$ at $p \in \mu^{-1}(0)$. Then, for each $Y \in \mathcal{G}_{p}$ we have

$$
g\left(X_{1}, Y\right)=g\left(I X_{1}, I Y\right)=g\left(I X_{1}+S X_{2}+T X_{3}, I Y\right)=0
$$

at $p$. Thus by condition (D), $X_{1}=0$. Similarly, $X_{2}=0=X_{3}$, and condition (S) holds.

For the second part, if condition (D) fails, then there is a $p \in \mu^{-1}(0)$ such that $V:=\left(\mathcal{G} \cap \mathcal{G}^{\perp}\right)_{p}$ is non-zero. Suppose $\omega_{I}^{\prime}$ is non-degenerate. Then ker $i^{*} \omega_{I}=$ $\operatorname{ker} \pi_{*} \omega_{I}^{\prime}=\mathcal{G}$. Equation (4.2) gives that $S V$ and $T V$ are subspaces of $\mathcal{G}$. However, $S V$ is contained in $S \mathcal{G}$ which is orthogonal to $\mathcal{G}$. Hence, $V$ is invariant under $S$ and $T$ and hence under $I=S T$. Taking $X_{1}=I X, X_{2}=S X, X_{3}=0$ for some nonzero $X$ in $V$, we see that condition (S) is violated. Thus if the quotient geometry is non-degenerate and (S) holds, then (D) must hold too.

A simple case of the above result is:

Corollary 4.7. A hypersymplectic quotient by a free circle action with Killing field $X$ is a smooth hypersymplectic manifold except at points where $g(X, X)=0$.

It will be useful, in light of Corollary 4.7 to have a formula for the length of the Killing field of a circle action on $\mathbb{C}^{d, d}$. If the action is given by

$$
\left(z_{k}, w_{k}\right) \longmapsto\left(e^{\mathbf{i} \theta_{k} t} z_{k}, e^{\mathbf{i} \theta_{k} t} w_{k}\right)
$$

then the associated vector field is

$$
X=\sum_{k=1}^{d} \mathbf{i} \theta_{k} z_{k} \frac{\partial}{\partial z_{k}}+\mathbf{i} \theta_{k} w_{k} \frac{\partial}{\partial w_{k}}-\mathbf{i} \theta_{k} \bar{z}_{k} \frac{\partial}{\partial \bar{z}_{k}}-\mathbf{i} \theta_{k} \bar{w}_{k} \frac{\partial}{\partial \bar{w}_{k}},
$$

giving

$$
g(X, X)=\sum_{k=1}^{d} \theta_{k}^{2}\left(\left|z_{k}\right|^{2}-\left|w_{k}\right|^{2}\right) .
$$

We next investigate when condition (S) of Definition 4.2 holds for general toric reductions of flat space. For three vector fields $X_{1}, X_{2}, X_{3}$ with coefficients $\theta_{k}^{(i)}$, 
$i=1,2,3$, 4.1 becomes

$$
\begin{aligned}
\theta_{k}^{(1)} z_{k} & =\left(\mathbf{i} \theta_{k}^{(2)}-\theta_{k}^{(3)}\right) w_{k}, & & \text { for } k=1, \ldots, d, \\
\theta_{k}^{(1)} w_{k} & =-\left(\mathbf{i} \theta_{k}^{(2)}+\theta_{k}^{(3)}\right) z_{k}, & & \text { for } k=1, \ldots, d .
\end{aligned}
$$

For each $k$, these equations have the form

$$
a z=b w, \quad a w=\bar{b} z,
$$

with $a \in \mathbb{R}$ and $b \in \mathbb{C}$. We deduce that $a^{2} z=a b w=|b|^{2} z$, and similarly $a^{2} w=$ $|b|^{2} w$. So the system (4.4) has a solution if only if for each $k$,

$$
\begin{aligned}
\text { either } & \left(z_{k}, w_{k}\right)=0, \\
\text { or } & \theta_{k}^{(1)}=0=\theta_{k}^{(2)}=\theta_{k}^{(3)}, \\
\text { or } & z_{k}=\xi_{k} w_{k} \text { and } \xi_{k} \theta_{k}^{(1)}=\mathbf{i}\left(\theta_{k}^{(2)}+\mathbf{i} \theta_{k}^{(3)}\right), \\
& \quad \text { for some } \xi_{k} \text { with }\left|\xi_{k}\right|=1 .
\end{aligned}
$$

We also discuss the question of degeneracy of the hypersymplectic structure. We know from Theorem 4.6 and the discussion leading up to (4.3) that degeneracy occurs if and only if the inner product

$$
q=\operatorname{diag}\left(\left|z_{k}\right|^{2}-\left|w_{k}\right|^{2}\right)_{k=1}^{d}
$$

is degenerate on $\mathfrak{n} \leqslant \mathbb{R}^{d}$ at some point $(z, w) \in \mu^{-1}(0)$. Equivalently, in the notation of $₫ 3$, at some point of $\mu^{-1}(0)$ there is $\zeta \in \mathfrak{n} \backslash\{0\}$ such that $q(\zeta, \cdot) \in \operatorname{ker} \iota^{*}=\operatorname{im} \beta^{*}$. From (3.3), this condition is equivalent to the existence of $\zeta \in \mathfrak{n} \backslash\{0\}$ and $s \in \mathbb{R}^{n}$ such that

$$
\zeta_{k}\left(\left|z_{k}\right|^{2}-\left|w_{k}\right|^{2}\right)=\left\langle s, u_{k}\right\rangle, \quad \text { for } k=1, \ldots, d .
$$

In $\$ 5$ we shall refine both of these criteria.

\section{TORIC GEOMETRY OF THE QUOTIENT}

We shall now study some properties of the hypersymplectic quotient $M$ of $\mathbb{C}^{d, d}$ by a compact Abelian group $N \subset \mathbb{T}^{d}$. This quotient carries an action of the torus $\mathbb{T}^{n}=\mathbb{T}^{d} / N$, and we may consider the map $\phi: M \rightarrow \mathbb{R}^{3 n}$ given by

$$
\phi:(z, w) \longmapsto(a, b),
$$

where $a$ and $b$ are as in (3.5) and (3.6). When $M$ is smooth and hypersymplectic this is the moment map for the action of $\mathbb{T}^{n}$ on $M$.

A similar map is considered in the Kähler and hyperkähler cases [Gu, $\mathrm{BD}$. In the first case, the analogue of $\phi$ is a map $M \rightarrow \mathbb{R}^{n}$ with image the polyhedron defined by (3.2). The map induces a homeomorphism of $M / \mathbb{T}^{n}$ onto the polyhedron.

In the hyperkähler case, one has a map onto the whole of $\mathbb{R}^{3 n}$, and again a homeomorphism $M / \mathbb{T}^{n} \cong \mathbb{R}^{3 n}$. Essentially, this follows from the $N=\{1\}$ case, i.e., the fact that the moment map $\left(z_{k}, w_{k}\right) \longmapsto\left(\frac{1}{2}\left(\left|z_{k}\right|^{2}-\left|w_{k}\right|^{2}\right), \mathbf{i} z_{k} w_{k}\right)$ for the hyperkähler action of $\mathbb{T}^{d}$ on $\mathbb{H}^{d}$ induces a homeomorphism from $\mathbb{H}^{d} / \mathbb{T}^{d}$ onto $\mathbb{R}^{3 d}$.

In our case, the image of $\phi$ is an interesting subset of $\mathbb{R}^{3 n}$, but we no longer obtain a homeomorphism. Indeed the fibres of $\phi$ may be disconnected. 
We introduce the following notation:

$$
\begin{aligned}
a_{k} & :=\left\langle a, u_{k}\right\rangle-\lambda_{k}^{(1)}, \\
b_{k} & :=\left\langle b, u_{k}\right\rangle-\lambda_{k}^{(c)},
\end{aligned}
$$

for $a \in \mathbb{R}^{n}, b \in \mathbb{C}^{n}, k=1, \ldots, d$, and where

$$
\lambda_{k}^{(c)}:=\lambda_{k}^{(2)}+\mathbf{i} \lambda_{k}^{(3)} .
$$

Proposition 5.1. The image of the moment map $\phi$, (5.1), is the set

$$
K=\left\{(a, b) \in \mathbb{R}^{n} \times \mathbb{C}^{n}: a_{k} \geqslant\left|b_{k}\right|, \text { for } k=1, \ldots, d\right\} .
$$

Moreover $\phi$ induces a finite-to-one map $\tilde{\phi}$ from $M / \mathbb{T}^{n}$ onto $K$. The fibre of $\tilde{\phi}$ over $(a, b)$ has $2^{m}$ points, where $m$ is the number of the inequalities in the definition of $K$ which are strict for $(a, b)$.

Proof. By (3.5) and (3.6),$(z, w)$ is in $\mu^{-1}(0) \subset \mathbb{C}^{d, d}$ and satisfies $\phi(z, w)=(a, b)$ if and only if

$$
\left|z_{k}\right|^{2}+\left|w_{k}\right|^{2}=2 a_{k} \quad \text { and } \quad z_{k} \bar{w}_{k}=-\mathbf{i} b_{k},
$$

for $k=1, \ldots, d$. The torus $\mathbb{T}^{d}$ acts by $\left(z_{k}, w_{k}\right) \mapsto\left(e^{\mathbf{i} \theta_{k}} z_{k}, e^{\mathbf{i} \theta_{k}} w_{k}\right)$. On $\mu^{-1}(0)$ we have that $\left|w_{k}\right|$ uniquely determines $\left(z_{k}, w_{k}\right)$ up to the action of $\mathbb{T}^{d}$. Taking the absolute value of the second equation and using the first equation to eliminate $\left|z_{k}\right|^{2}$, we get

$$
\left|w_{k}\right|^{2}=a_{k} \pm \sqrt{a_{k}^{2}-\left|b_{k}\right|^{2}} .
$$

Thus there is a solution for $\left|w_{k}\right|^{2}$ only if $a_{k} \geqslant\left|b_{k}\right|$. There are two solutions if the inequality is strict, otherwise there is only one solution. The result follows.

Remark 5.2. Taking $d=1$ and $N=\{1\}$ in the proof of the proposition, $\phi$ is the hypersymplectic moment map for the action of $\mathbb{T}^{1}$ on $\mathbb{C}^{1,1}$. One can see that the corresponding hypersymplectic quotient may be two points, a single point, or empty depending on the choice of level set. So we may get smooth quotient sets of different topology for different choices of level set, contrasting with the hyperkähler case.

Proposition 5.3. The set $K=\phi(M)$ is convex in $\mathbb{R}^{3 n}$.

Proof. Using (5.2), the set $K$ is the intersection of sets

$$
K_{k}=\left\{(a, b) \in \mathbb{R}^{n} \times \mathbb{C}^{n}: a_{k} \geqslant\left|b_{k}\right|\right\}, \quad k=1, \ldots, d .
$$

However, $K_{k}$ is the preimage of the solid cone $\{(x, z) \in \mathbb{R} \times \mathbb{C}: x \geqslant|z|\}$ under the affine map $(a, b) \mapsto\left(a_{k}, b_{k}\right)$. Thus $K_{k}$ is convex, and it follows that $K$ is convex, too.

Convexity implies in particular that $K$ is connected, and Proposition 5.1 now gives:

Corollary 5.4. $M$ is connected if and only if each inequality in the definition of $K$ is an equality at some point of $K$.

Theorem 5.5. The hypersymplectic quotient $M=\mu^{-1}(0) / N$ is compact if and only if the vectors $u_{1}, \ldots, u_{d}$ define a bounded polyhedron in $\mathbb{R}^{n}$. 


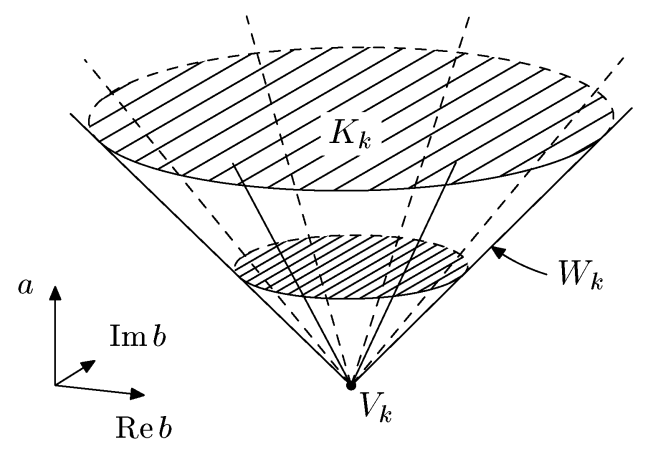

Figure 5.1. The solid cone $K_{k}$ when $n=1$.

Proof. Suppose $u_{1}, \ldots, u_{d}$ define a bounded polyhedron. As

$$
M=\left(\mu_{I}^{-1}(0) \cap\left(\mu_{S}+\mathbf{i} \mu_{T}\right)^{-1}(0)\right) / N,
$$

it is enough to show compactness of $\mu_{I}^{-1}(0)$.

If $(z, w) \in \mu_{I}^{-1}(0)$, then the vector $a$ of (3.5) must live in the polyhedron $\{a$ : $\left.\left\langle a, u_{k}\right\rangle \geqslant \lambda_{k}^{(1)}\right\}$, which is compact by hypothesis. Now (3.5) gives us a bound on the $\left|z_{k}\right|$ and $\left|w_{k}\right|$ in terms of the $u_{k}$ and $\lambda_{k}$.

Conversely, note that $M$ is compact if and only if $M / \mathbb{T}^{n}$ is compact, and hence if and only if $K$ is compact. We may define a projection $p: K \rightarrow \mathbb{C}^{n}$ by $p(a, b)=b$. The fibres of $p$ are the polyhedra

$$
F_{b}=\left\{a \in \mathbb{R}^{n}:\left\langle a, u_{k}\right\rangle \geqslant \lambda_{k}^{(1)}+\left|b_{k}\right|\right\} .
$$

If $M$ is compact, then $F_{b}$ is compact for each $b \in p(K)$. As $F_{b}$ is non-empty, this implies that $u_{1}, \ldots, u_{d}$ define a bounded polyhedron as in (3.2).

As promised in 4 , we now refine the criteria for smoothness and non-degeneracy of the hypersymplectic quotient $M$, in terms of the map $\phi$ and the vectors $u_{k}$.

In order to consider smoothness of $M$, we need to discuss the orbit types of the actions of $N$ on $\mu^{-1}(0)$ and the smoothness of $\mu^{-1}(0)$ itself. Let us begin with the orbit types. In the notation (5.2), put

$$
V_{k}:=\left\{(a, b) \in \mathbb{R}^{n} \times \mathbb{C}^{n}: a_{k}=0=b_{k}\right\} ;
$$

see Figure 5.1. Note that (3.5) and (3.6) show that for $(z, w) \in \mu^{-1}(0)$

$$
z_{k}=w_{k}=0 \quad \text { if and only if } \quad(a, b)=\phi(z, w) \in V_{k} .
$$

Following [Gu], if $A$ is a subset of $\{1, \ldots, d\}$ we denote by $\mathbb{T}_{A}$ the torus whose Lie algebra is

$$
\mathfrak{t}_{A}=\operatorname{span}_{\mathbb{R}}\left\{e_{k}: k \in A\right\} .
$$

We deduce that the stabiliser of $(z, w)$, for the $\mathbb{T}^{d}$ action, is $\mathbb{T}_{J}$, where

$$
J:=\left\{k: \phi(z, w) \in V_{k}\right\} .
$$

Proposition 5.6. For $(z, w) \in \mu^{-1}(0)$ :

(i) $\operatorname{Stab}_{N}(z, w)$ equals $\mathbb{T}_{J} \cap N$, where $\mathbb{T}_{J}$ is the torus whose Lie algebra is spanned by the vectors $e_{k}$ for which $\phi(z, w) \in V_{k}$;

(ii) on $M, \operatorname{Stab}_{\mathbb{T}^{n}}(z, w)$ is the torus whose Lie algebra is spanned by the vectors $u_{k}$ such that $\phi(z, w) \in V_{k}$. 
Moreover, putting $\mathcal{B}_{x}=\left(u_{k}: x \in V_{k}\right)$,

(iii) $\operatorname{Stab}_{N}(z, w)$ is finite for all $(z, w) \in \mu^{-1}(0)$ if and only if for each $x \in \phi(M)$, the collection of vectors $\mathcal{B}_{x}$ is linearly independent;

(iv) $\operatorname{Stab}_{N}(z, w)=1$ for all $(z, w) \in \mu^{-1}(0)$ if and only if for each $x \in \phi(M)$, the collection of vectors $\mathcal{B}_{x}$ is contained in a $\mathbb{Z}$-basis for $\mathbb{Z}^{n}$.

Proof. Statements (i) and (ii) follow from the above discussion, while (iii) and (iv) follow from (i), (ii) and results of Delzant [De] and Guillemin $[\mathrm{Gu}$, as cited in the proof of Theorems 3.2 and 3.3 in $\mathrm{BD}$. To see this, note that $V_{k}$ is the affine flat $H_{k}$ introduced in $\mathrm{BD}$.

Remark 5.7. The argument of Theorems 3.2 and 3.3 of [BD] shows that if every $n+1$ of the $V_{k}$ have empty intersection, then the condition of (iii) holds and hence $N$ acts locally freely on $\mu^{-1}(0)$. In particular, for any given collection of vectors $u_{k}$, the action will be locally free for generic choice of $\lambda$. Hence, by Sard's theorem, $M=\mu^{-1}(0) / N$ will have at worst orbifold singularities for generic choice of $\lambda$.

Similarly, we see that if for each $A \subset\{1, \ldots, d\}$ of size $n$, the collection $\left(u_{k}: k \in\right.$ $A$ ) is a $\mathbb{Z}$-basis for $\mathbb{Z}^{n}$, then $M=\mu^{-1}(0) / N$ is a manifold for generic $\lambda$.

To determine precise conditions for the smoothness of $\mu^{-1}(0)$, let

$$
W_{k}:=\left\{(a, b) \in \mathbb{R}^{n} \times \mathbb{C}^{n}: a_{k}=\left|b_{k}\right|\right\} .
$$

A point $(z, w) \in \mu^{-1}(0)$ has $\phi(z, w) \in W_{k}$ if and only if $\left|z_{k}\right|=\left|w_{k}\right|$. Note that $V_{k} \subset W_{k}$ and that both sets may be empty for a given $k$. Let $J$ be as in (5.4) above and put

Note that $L$ contains $J$

$$
L:=\left\{\ell: \phi(z, w) \in W_{\ell}\right\}
$$

Consider the equations (4.5) at $(z, w) \in \mu^{-1}(0)$. For $k \in J$, there is no restriction on $\theta_{k}^{(i)}$. For $k \in L^{\prime}$, the complement of $L$ in $\{1, \ldots, d\}$, we require $\theta_{k}^{(i)}=0$, for $i=1,2,3$. For $k \in L \backslash J$, we have $\xi_{k} \theta_{k}^{(1)}=\mathbf{i}\left(\theta_{k}^{(2)}+\mathbf{i} \theta_{k}^{(3)}\right)$. For such $k$, we have $\left|z_{k}\right|=\left|w_{k}\right| \neq 0$, so $\xi_{k}$ is uniquely determined by

$$
\xi_{k}=\frac{z_{k}}{w_{k}}=\frac{z_{k} \bar{w}_{k}}{\left|w_{k}\right|^{2}}=-\mathbf{i} \frac{b_{k}}{a_{k}} .
$$

Consider the map

$$
\begin{gathered}
\Lambda_{(a, b)}: \mathfrak{n}_{L, J} \otimes(\mathbb{R} \times \mathbb{C}) \rightarrow \mathbb{C}_{L \backslash J}, \\
\Lambda_{(a, b)}\left(\theta_{k}^{(1)}, \theta_{k}^{(c)}\right)=\left(b_{k} \theta_{k}^{(1)}+a_{k} \theta_{k}^{(c)}\right),
\end{gathered}
$$

where $\mathfrak{n}_{L, J}$ is the projection to $\mathfrak{t}_{J}$ of $\mathfrak{n}_{L}:=\mathfrak{n} \cap \mathfrak{t}_{L}$. Alternatively, $\mathfrak{n}_{L, J}$ is the kernel of the map $\beta_{L, J}: \mathfrak{t}_{L \backslash J} \rightarrow \mathbb{R}^{n} / \operatorname{im}\left(\left.\beta\right|_{\mathfrak{t}_{J}}\right)$ induced by $\beta$.

Proposition 5.8. For a locally free action of $N$ on $\mu^{-1}(0)$, condition (S) of Definition 4.2 for smoothness of the level set $\mu^{-1}(0)$ holds if and only if the linear map $\Lambda_{(a, b)}$ of (5.5) is injective at each point $(a, b)$ of $K$.

Remark 5.9. Over the combinatorial interior of $K$,

$$
\operatorname{CInt}(K)=K \backslash \bigcup_{k=1}^{d} W_{k},
$$

the set $L$ is empty, so $\phi^{-1}$ (CInt $\left.K\right) \subset M$ is always a smooth manifold. The combinatorial interior is equal to the topological interior if $K_{k} \neq W_{k}$, for all $k$. 
Remark 5.10. By Proposition 5.6, if the action of $N$ is locally free, then $\mathfrak{n}_{L}$ is transverse to $\mathfrak{t}_{J}$ and hence the orthogonal projection $\mathfrak{n}_{L} \rightarrow \mathfrak{n}_{L, J}$ is injective. Thus the smoothness condition will necessarily fail if $3 \operatorname{dim} \mathfrak{n}_{L}>2|L \backslash J|$. Since $\operatorname{dim} \mathfrak{n}_{L} \geqslant$ $|L|-n$, we conclude that smoothness requires $|L| \leqslant 3 n$, i.e., no more than $3 n$ of the $W_{k}$ 's may meet in $K$.

Finally, let us consider non-degeneracy of the hypersymplectic structure.

Proposition 5.11. The non-degeneracy condition (D) of Definition 4.5 fails at some point $(z, w) \in \mu^{-1}(0)$ with $\phi(z, w)=(a, b)$ if and only if there exist scalars $\zeta_{1}, \ldots, \zeta_{d}$, not all zero, and $s \in \mathbb{R}^{n}$ such that

$$
4 \zeta_{k}^{2}\left(a_{k}^{2}-\left|b_{k}\right|^{2}\right)=\left\langle s, u_{k}\right\rangle^{2}, \quad \text { for } k=1, \ldots, d
$$

and

$$
\sum_{k=1}^{d} \zeta_{k} u_{k}=0
$$

Proof. This is immediate from the the discussion at the end of 4 , equation (4.7), the definition of $\mathfrak{n}$ as $\operatorname{ker} \beta$, and the proof of Proposition 5.1 which expresses $\left|z_{k}\right|$ and $\left|w_{k}\right|$ in terms of $a$ and $b$.

Remark 5.12. Consider the special case when $s=0$. The expression $a_{k}{ }^{2}-\left|b_{k}\right|^{2}$ is zero on $W_{k}$. Suppose $A \subset\{1, \ldots, d\}$ has $n+1$ elements. Then $\left(u_{k}: k \in A\right)$ is linearly dependent, so we may find $\left(\zeta_{1}, \ldots, \zeta_{d}\right) \in \mathfrak{t}_{A} \backslash\{0\}$ satisfying (5.7). If

$$
K \cap \bigcap_{k \in A} W_{k} \neq \varnothing
$$

we then obtain a solution to the remaining equations (5.6). Thus if $n+1$ of the $W_{k}$ 's meet in $K$, then condition (D) necessarily fails. This is a considerably stronger restriction than that obtained for smoothness in Remark 5.10, and as we will see in 97 , one may easily obtain smooth quotients with hypersymplectic structures that degenerate on some hypersurface.

Theorem 5.13. Let $\phi$ be the moment map for the action of $\mathbb{T}^{n}$ on the hypersymplectic quotient $M=\mu^{-1}(0) / N$. Suppose the combinatorial interior $\operatorname{CInt}(K)$ of the image $K$ of $\phi$ is non-empty. Then an open subset of $M$ carries a smooth non-degenerate hypersymplectic structure.

When $M$ is compact, the degeneracy locus is non-empty of codimension at least one.

Non-trivial examples of quotients with empty degeneracy locus will be given in 87 .

Proof. We have already noted that $M$ is smooth over $\operatorname{CInt}(K)$. For $(a, b) \in$ $\operatorname{CInt}(K)$, we have $a_{k}^{2} \neq\left|b_{k}\right|^{2}$ for all $k$. Equations (5.6) may thus be solved for $\zeta_{k}$ and (5.7) becomes

$$
H(s, w):=\sum_{k=1}^{d} w_{k}\left\langle s, u_{k}\right\rangle u_{k}=0
$$


with $w=\left(w_{1}, \ldots, w_{d}\right), w_{k}=1 /\left(2 \varepsilon_{k} \sqrt{a_{k}^{2}-\left|b_{k}\right|^{2}}\right), \varepsilon_{k}= \pm 1$ and $s \neq 0$. Now $H: R_{1} \times R_{2} \rightarrow \mathbb{R}^{n}$, where $R_{1}=\mathbb{R}^{n} \backslash\{0\}$ and $R_{2}$ is an $n$-dimensional manifold contained in the complement of the coordinate axes in $\mathbb{R}^{d}$, has differential $D H_{(s, w)}(\varsigma, v)=\sum_{k=1}^{d}\left\langle w_{k} \varsigma+v_{k} s, u_{k}\right\rangle u_{k}$ which has rank $n$ when $w_{k} \neq 0$ for all $k$. Thus $H^{-1}(0)$ is a submanifold of $R_{1} \times R_{2} \subset \mathbb{R}^{n} \times \mathbb{R}^{d}$ of dimension $n$. But if $(s, w)$ lies in $H^{-1}(0)$, then so does $(\lambda s, w)$ for $\lambda \in \mathbb{R} \backslash 0$. So the projection of $H^{-1}(0)$ to the second factor $R_{2}$, has dimension at most $n-1$, i.e., the degeneracy locus is at least codimension one.

Now suppose that $M$ is compact. Consider the map $p: K \rightarrow \mathbb{C}^{n}$ of Theorem 5.5 and its fibres $F_{b}$. Note that when the interior Int $F_{b}$ is non-empty we have Int $F_{b}=$ $\left\{a \in \mathbb{R}^{n}: a_{k}>\left|b_{k}\right|\right\}$. As this is an open condition on $b$, we see that $p\left(\operatorname{Int} F_{b}\right) \subset$ $\operatorname{Int}(p(K))$. Fix $b$ on the boundary of $p(K)$. Then $F_{b}$ is a compact polytope in $\mathbb{R}^{n}$ with empty interior. Let $v$ be a vertex of that polytope. Then $v$ is at the intersection of at least $n$ hyperplanes $a_{k}=\left|b_{k}\right|$. However, if only $n$ hyperplanes meet, then we can find interior points of $F_{b}$ close to $v$. Thus $n+1$ hyperplanes meet in $v$. This is the same as saying that $(v, b)$ lies on $n+1$ of the $W_{k}$. By Remark 5.12, this implies that the hypersymplectic structure is degenerate at $(v, b)$.

We shall next relate our quotients to toric varieties. We first prove a lemma.

Lemma 5.14. Let $\delta: \mathbb{T}^{d} \rightarrow \mathbb{T}^{d} \times \mathbb{T}^{d}=\mathbb{T}^{2 d}$ be the diagonal map, and let $N$ be a compact Abelian subgroup of $\mathbb{T}^{d}$.

If $N \leqslant \mathbb{T}^{d}$ is defined by a collection of vectors in $\mathbb{R}^{n}$ defining a bounded polyhedron, then $\delta(N) \leqslant \mathbb{T}^{2 d}$ is defined by a collection of vectors in $\mathbb{R}^{n+d}$ defining a bounded polyhedron.

Proof. We have an exact sequence

$$
0 \longrightarrow \delta_{*}(\mathfrak{n}) \stackrel{\tilde{\iota}}{\longrightarrow} \mathbb{R}^{d} \oplus \mathbb{R}^{d}=\mathbb{R}^{2 d} \stackrel{\tilde{\beta}}{\longrightarrow} \mathbb{R}^{n+d} \longrightarrow 0,
$$

for some map $\tilde{\beta}$. It is straightforward to check that $\delta_{*}(\mathfrak{n})=\{(v, v): v \in \mathfrak{n}\}$ is the kernel of the map defined by

$$
e_{k} \longmapsto \tilde{u}_{k}:= \begin{cases}u_{k}+e_{k+d}, & \text { if } k \leqslant d, \\ -e_{k}, & \text { if } k>d .\end{cases}
$$

So we can take this map to be $\tilde{\beta}$, and hence $\delta_{*}(\mathfrak{n})$ is defined by vectors $\tilde{u}_{1}, \ldots, \tilde{u}_{2 d}$.

Consider the polyhedron $\left\{s \in \mathbb{R}^{n+d}:\left\langle s, \tilde{u}_{k}\right\rangle \geqslant c_{k}, k=1, \ldots, 2 d\right\}$. The defining inequalities may be written as

$$
\begin{aligned}
\left\langle s_{1}, u_{k}\right\rangle+\left\langle s_{2}, e_{k+d}\right\rangle & \geqslant c_{k}, & & \text { for } k=1, \ldots, d, \\
\left\langle s_{2},-e_{k+d}\right\rangle & \geqslant c_{k+d}, & & \text { for } k=1, \ldots, d,
\end{aligned}
$$

where $s=s_{1}+s_{2}, s_{1} \in \mathbb{R}^{n}$ and $s_{2} \in \operatorname{span}\left\{e_{d+1}, \ldots, e_{2 d}\right\}$. We see that $\left\langle s_{1}, u_{k}\right\rangle \geqslant c_{k}+$ $c_{k+d}$, for $k=1, \ldots, d$. As $u_{1}, \ldots, u_{d}$ define a bounded polyhedron by hypothesis, we get a bound on $s_{1}$, and hence, from

$$
c_{k}-\left\langle s_{1}, u_{k}\right\rangle \leqslant\left\langle s_{2}, e_{k+d}\right\rangle \leqslant-c_{k+d}
$$

a bound on $s_{2}$.

Now, observe that $\mu_{I}$ is just the moment map $m$ for the Kähler action of $\delta(N)$ on $\mathbb{C}^{2 d}$. (Here $\mathbb{C}^{2 d}$ has the standard complex structure $I_{0}$, not the hypersymplectic complex structure $I$, as remarked in $\$ 2$, ) 
The construction of Guillemin and Delzant now gives:

Theorem 5.15. $M$ is the sub-variety

$$
\mu_{S}+\mathbf{i} \mu_{T}=0
$$

in the toric variety $m^{-1}(0) / \delta(N)$. If the vectors $u_{k}$ define a bounded polyhedron, then by Lemma 5.14 this toric variety is compact.

Remark 5.16. Note that $\mu_{S}+\mathbf{i} \mu_{T}$, although $I$-holomorphic, is not holomorphic with respect to the complex structure on this toric variety, which is induced from $I_{0}$.

We conclude by discussing two actions that can occur on $M$ for special choices of $\lambda$.

Remark 5.17. If we take $\lambda_{k}^{(j)}=0$ for all $j, k$, then $(0,0) \in \mu^{-1}(0)$ and is a fixed point of $N$, giving a singular point in the quotient $M=\mu^{-1}(0) / N$. In fact it follows from (3.5), (3.6) with $\lambda_{j}^{(k)}=0$ that we have a scaling action $(z, w) \mapsto(t . z, t . w)$, for $t \in \mathbb{R}^{*}$ on $\mu^{-1}(0)$ which descends to the quotient, so that $M$ is a cone with vertex at $(0,0)$.

Remark 5.18. Harada and Proudfoot $\mathrm{HP}$ have observed that in the hyperkähler case, if we take $\lambda_{k}^{(2)}+\mathbf{i} \lambda_{k}^{(3)}=0$ for all $k$, then the quotient $M$ admits a circle action

$$
(z, w) \mapsto\left(z, e^{\mathbf{i} \psi} w\right)
$$

This action is holomorphic with respect to $I$ but not with respect to $J$ or $K$.

This action does not occur for the Kähler quotients of $\mathrm{Gu}$, De. However we observe that it does exist for our hypersymplectic quotients, provided we take $\lambda_{k}^{(2)}+$ $\mathbf{i} \lambda_{k}^{(3)}=0$ as above. The action is compatible with $I$ but not with $S$ or $T$.

Note also that under this condition on $\lambda$, the hypersymplectic quotient contains two distinguished subvarieties, defined by the vanishing of $z$ and $w$, respectively. Each of these may be identified with a Kähler quotient of $\mathbb{C}^{d}$ by $N$, and hence with a toric variety.

The locus $w=0$ lies in the fixed point set of the circle action. As $M$ is a quotient, we may in addition have other components of the fixed point set. We find, as in the hyperkähler case $[\mathrm{HP}$, that the fixed point set in general is a union of toric varieties which may be enumerated in terms of conditions on the vectors $u_{k}$.

To make this precise, for $A, B \subset\{1, \ldots, d\}$, let

$$
\begin{aligned}
& M_{A, B}=\left\{[z, w] \in M: z_{k}=0 \text { for all } k \in A\right. \\
& \text { and } \left.w_{\ell}=0 \text { for all } \ell \in B\right\} .
\end{aligned}
$$

Note that $M_{A, A^{\prime}}$ is a toric variety. The fixed point condition

$$
\left(z, e^{i \psi} w\right)=(g(\psi) z, g(\psi) w)
$$

for $g(\psi) \in N$ implies immediately that $w_{k}=0$ whenever $z_{k} \neq 0$. Further examination of this condition leads to:

Proposition 5.19. When $\lambda_{k}^{(2)}+\mathbf{i} \lambda_{k}^{(3)}=0$, the hypersymplectic quotient $M$ admits a circle action with fixed point set the union of the toric varieties $M_{A, B}$ where $A \cup B=\{1, \ldots, d\}$ and

$$
\sum_{k \in A} u_{k} \in \operatorname{span}\left\{u_{\ell}: \ell \in A \cap B\right\} .
$$




\section{InVOLUTiOnS}

In the Kähler case studied by Guillemin and Delzant, the moment map $\mu: \mathbb{C}^{d} \mapsto$ $\mathfrak{n}^{*}$ is invariant under the involution of $\mathbb{C}^{d}$ given by complex conjugation. In fact, conjugation induces an involution $\gamma$ of the quotient $M=\mu^{-1}(0) / N$. Moreover the fixed point set of complex conjugation in $\mu^{-1}(0)$ is a cover of the fixed point set of $\gamma$ in $M$. The group of deck transformations is the finite group $\Gamma$ of involutions in $N$.

In the hyperkähler situation there appears to be no such involution in general.

In our hypersymplectic case, however, we do have an involution. Explicitly, the $\operatorname{map} \sigma: \mathbb{C}^{d, d} \mapsto \mathbb{C}^{d, d}$ given by

$$
\sigma:\left(z_{k}, w_{k}\right) \mapsto\left(\bar{w}_{k}, \bar{z}_{k}\right)
$$

preserves $\mu^{-1}(0)$ and sends $N$-orbits to $N$-orbits. (It does not commute with the action, but we have $\sigma(g \cdot(z, w))=g^{-1} \cdot \sigma(z, w)$, which suffices.)

It follows that $\sigma$ induces an involution $\hat{\sigma}$ on the hypersymplectic quotient $M=$ $\mu^{-1}(0) / N$. Let us denote by $\mu^{-1}(0)_{\sigma}$ and $M_{\hat{\sigma}}$ the fixed point sets of the involutions in $\mu^{-1}(0)$ and $M$, respectively.

Theorem 6.1. The natural projection $\pi: \mu^{-1}(0) \rightarrow M$ induces a surjection

$$
\mu^{-1}(0)_{\sigma} \rightarrow M_{\hat{\sigma}}
$$

If $N$ acts freely on $\mu^{-1}(0)$ this map is a cover whose group of deck transformations is the finite group

$$
\Gamma=\left\{h \in N: h^{2}=1\right\}
$$

Proof. Observe first that $(z, w) \in \mu^{-1}(0)$ represents a point in $M_{\hat{\sigma}}$ if and only if there exists $g \in N$ with $w=g^{-1} \bar{z}=\overline{g z}$. Now if $h \in N$ satisfies $h^{2}=g$, then

$$
h \cdot(z, w)=h \cdot(z, \overline{g z})=\left(h z, \overline{h^{-1} g z}\right)=(h z, \overline{h z}),
$$

so $(z, w)$ represents the same point in $M$ as does $(h z, \overline{h z}) \in \mu^{-1}(0)_{\sigma}$. This proves the surjectivity assertion.

Next, suppose that two points $(z, \bar{z})$ and $(u, \bar{u})$ in $\mu^{-1}(0)_{\sigma}$ are related by the action of $g \in N$. We need, for each $k$,

$$
z_{k}=e^{\mathbf{i} \theta_{k}} v_{k} \quad \text { and } \quad \bar{z}_{k}=e^{\mathbf{i} \theta_{k}} \bar{v}_{k}
$$

for some $e^{\mathbf{i} \theta_{k}} \in \mathbb{T}^{1}$. Hence either $e^{i \theta_{k}}$ is an order two element in $\mathbb{T}^{1}$ or $z_{k}=v_{k}=0$. We deduce that $g^{2} \in \mathbb{T}_{J} \cap N$, where $J$ is the set of indices for which $z_{k}=0$.

If $N$ acts freely on $\mu^{-1}(0)$, then $\mathbb{T}_{J} \cap N$ is trivial by Proposition 5.6. The remaining assertions now follow easily.

Proposition 6.2. The symplectic forms $\omega_{I}, \omega_{S}$ and $\omega_{T}$ all vanish when restricted to $M_{\hat{\sigma}}$.

Proof. The involution $\hat{\sigma}$ pulls back $\omega_{I}, \omega_{S}$ and $\omega_{T}$ to their negatives.

Remark 6.3. Non-degenerate hypersymplectic manifolds with multi-Lagrangian subsets have recently appeared in [FPPW]. There hypersymplectic structures arise on $\mathbb{T}^{2 n}$-fibrations over a $\mathbb{T}^{2 n}$ base, and the fibres are multi-Lagrangian. 
In contrast, we have:

Proposition 6.4. The fixed point set $M_{\hat{\sigma}}$ is non-empty only if

$$
\bigcap_{k=1}^{d} W_{k} \neq \varnothing
$$

The non-degeneracy statement (D), Definition 4.5, is not satisfied on $\left.T M\right|_{M_{\hat{\sigma}}}$. In particular, if the action satisfies conditions $(\mathrm{F})$ and $(\mathrm{S})$ of Definitions 4.1 and 4.2, then $M$ is smooth but the hypersymplectic structure is degenerate over $M_{\hat{\sigma}}$.

Proof. If $(z, w) \in \mu^{-1}(0)$ is a fixed point for $\sigma$, then $\left|z_{k}\right|=\left|w_{k}\right|$ for $k=1, \ldots, d$. This is the condition that $\phi(z, w) \in W_{k}$ for $k=1, \ldots, d$, and the assertion follows.

When $\left|z_{k}\right|=\left|w_{k}\right|$ for all $k$, the quadratic form (4.6) is identically zero. Thus condition (D) fails. The final statement follows from Theorems 4.3 and 4.6 .

\section{EXAMPLES}

7.1. The diagonal circle action. Let us take $d=n+1$, and take

$$
\begin{gathered}
u_{k}=e_{k}, \quad \text { for } k=1, \ldots, n, \\
u_{n+1}=-\left(e_{1}+\cdots+e_{n}\right),
\end{gathered}
$$

so the vectors $u_{k}$ define the standard simplex in $\mathbb{R}^{n}$. Now $N$ is the standard diagonal circle in $\mathbb{T}^{n+1}$. The corresponding toric hyperkähler manifold is the Calabi space $T^{*} \mathbb{C} P(n)$.

In the hypersymplectic case $M=\mu^{-1}(0) / N$ is the quotient of the subset of $\mathbb{C}^{n+1, n+1}$ cut out by the equations

$$
\begin{aligned}
& \sum_{k=1}^{n+1}\left|z_{k}\right|^{2}+\left|w_{k}\right|^{2}=-2 \sum_{k=1}^{n+1} \lambda_{k}^{(1)}, \\
& \sum_{k=1}^{n+1} z_{k} \bar{w}_{k}=-\mathbf{i} \sum_{k=1}^{n+1}\left(\lambda_{k}^{(2)}+\mathbf{i} \lambda_{k}^{(3)}\right),
\end{aligned}
$$

by the action

$$
z_{k} \longmapsto e^{\mathbf{i} \theta} z_{k}, \quad w_{k} \longmapsto e^{\mathbf{i} \theta} w_{k}
$$

Using Theorem 5.15, this may be identified with a hypersurface in $\mu_{I}^{-1}(0) / N=$ $\mathbb{C P}^{2 n+1}$.

Note that the action

$$
(z, w) \longmapsto(A z, A w), \quad \text { for } A \in U(n+1)
$$

preserves the level set of $\mu^{-1}(0)$ and commutes with the action of $N$, so defines an effective $P U(n+1)$ action on $M$.

The stabiliser of $(z, w)$ is $P(U(1) \times U(n))$ if $z, w$ are linearly dependent, and $P(U(1) \times U(n-1))$ otherwise: the $P U(n+1)$ action on $M$ is therefore cohomogeneity one.

Let us write

$$
P=-2 \sum_{k=1}^{n+1} \lambda_{k}^{(1)}, \quad Q=-\mathbf{i} \sum_{k=1}^{n+1}\left(\lambda_{k}^{(2)}+\mathbf{i} \lambda_{k}^{(3)}\right) .
$$

A necessary condition for $M$ to be non-empty is $|Q| \leqslant \frac{1}{2} P$. 
The vector field $X$ for the action of $N$ takes the value $(\mathbf{i} z, \mathbf{i} w)$ at the point $(z, w)$. From $\$ 4$ we see that $I X, S X, T X$ are linearly independent unless $w=\lambda z$ for a complex number $\lambda$ of unit modulus. It is easy to check that such a point $(z, w)$ cannot lie in $\mu^{-1}(0)$ except in the special case $|Q|=\frac{1}{2} P$. We deduce that $M$ is a smooth manifold unless $|Q|=\frac{1}{2} P$.

The hypersymplectic structure on $M$ will degenerate at some points, however. Observe that the point $(z, w)$, where

$$
z=\left(\sqrt{\frac{1}{2} P}, 0, \ldots, 0\right), \quad w=\left(\frac{\bar{Q}}{\sqrt{\frac{1}{2} P}}, w_{2}, \ldots, w_{n+1}\right)
$$

and

$$
\sum_{k=2}^{n+1}\left|w_{k}\right|^{2}=\frac{1}{2} P-\frac{|Q|^{2}}{\frac{1}{2} P}
$$

lies in $\mu^{-1}(0)$ when this set is non-empty. Moreover, from (4.3), we have that the Killing field for the circle $N$ is null at $(z, w)$. Hence the hypersymplectic structure degenerates at this point.

If we take $Q=0$, then we have a circle action

$$
(z, w) \mapsto\left(z, e^{\mathbf{i} \psi} w\right)
$$

as discussed in Remark 5.18. The fixed point set of this action in $M=\mu^{-1}(0) / N$ is the union of the loci $z=0$ and $w=0$, since in Proposition 5.19 we have $A=\varnothing$ or $\{1, \ldots, n+1\}$. These fixed point sets are both diffeomorphic to $\mathbb{C P}^{n}$, and are the special orbits of the $P U(n+1)$ action in this case.

7.2. Codimension-one subgroups. Take $n=1$, and first consider

$$
u_{k}=e_{1}, \quad \text { for } k=1, \ldots, d,
$$

so

$$
\mathfrak{n}=\left\{\sum_{k=1}^{d} a_{k} e_{k}: \sum_{k=1}^{d} a_{k}=0\right\}
$$

and $N$ is the torus $\mathbb{T}^{d-1}=\left\{\left(t_{1}, \ldots, t_{d}\right): t_{1} t_{2} \ldots t_{d}=1\right\}$ in $\mathbb{T}^{d}$. The GibbonsHawking multi-instanton metrics are obtained as hyperkähler quotients of $\mathbb{H}^{d}$ by $N$.

We obtain, analogously, 4-dimensional hypersymplectic quotients of $\mathbb{C}^{d, d}$ by $N$, with an action of the circle group $\mathbb{T}^{d} / N$. Like the multi-instanton spaces, these hypersymplectic spaces are non-compact, as can be seen from Theorem 5.5.

Note that the sets $V_{k}$ of (5.3) are now just the $d$ points

$$
V_{k}=\left\{\left(\lambda_{k}^{(1)}, \lambda_{k}^{(2)}, \lambda_{k}^{(3)}\right)\right\}, \quad \text { for } k=1, \ldots, d .
$$

Proposition 5.6(iv) shows that $N$ acts freely on $\mu^{-1}(0)$ provided that these $d$ points are distinct (as in the hyperkähler case).

The set $K=\phi(M)$ in Proposition 5.1 is the intersection of the $d$ cones

$$
\left\{(a, b) \in \mathbb{R} \times \mathbb{C}: a-\lambda_{k}^{(1)} \geqslant\left|b-\left(\lambda_{k}^{(2)}+\mathbf{i} \lambda_{k}^{(3)}\right)\right|\right\}
$$

with vertices at $\left(\lambda_{k}^{(1)}, \lambda_{k}^{(2)}, \lambda_{k}^{(3)}\right)$, for $k=1, \ldots, d$. All these cones have the same angle and parallel axes.

The fixed points of the circle action correspond, from Proposition 5.6(ii), to the preimages under $\phi$ of those points $\left(\lambda_{k}^{(1)}, \lambda_{k}^{(2)}, \lambda_{k}^{(3)}\right)$ which lie in the intersection of the cones. 
We deduce that different configurations of the cones may give a zero or non-zero number of fixed points. This gives an example of different choices of level set giving quotient sets which are inequivalent as $\mathbb{T}^{n}$-manifolds.

By contrast, in the hyperkähler case, $\phi(M)$ is the whole of $\mathbb{R}^{3}$, and we always get $d$ fixed points for the circle action (provided that the points $H_{k}=V_{k}$ are distinct).

Let us now consider what may happen for arbitrary choices of $u_{k}$ when $n=1$ and $d$ is small.

Example 7.1. Consider $n=d=1$. This case is relatively trivial as the group $N$ is discrete. However, it illustrates a number of features of our constructions.

The map $\beta$ is defined by a single $u_{1} \in \mathbb{Z} \backslash\{0\}$, which we may take to be positive. The image of the moment map $\phi$ is the solid cone $K=K_{1}$ consisting of $(a, b) \in \mathbb{R} \times \mathbb{C}$ such that $a-\lambda_{1}^{(1)} / u_{1} \geqslant\left|b-\lambda_{1}^{(c)} / u_{1}\right|$, as in Figure 5.1. The vertex $V_{k}$ is the point $\left(\lambda_{1}^{(1)} / u_{1}, \lambda_{1}^{(c)} / u_{1}\right)$. The $N$-action is free only if $u_{1}=1$, in which case $N=\{1\}$. As $\mathfrak{n}=\{0\}$, the maps $\Lambda_{(a, b)}$ (5.5) are injective, so the quotient is indeed smooth when $u_{1}=1$. The equation (5.7) implies $\zeta_{1}=0$, confirming that the quotient geometry is non-degenerate.

In the case $u_{1}=1$, the induced map $\tilde{\phi}: \mathbb{C}^{1,1} / \mathbb{T}^{1} \rightarrow K=K_{1}$ is 2-to-1, branched over $\partial K=W_{1}$. Each $\operatorname{disc} \mathbb{D}(r)=\left\{(a, b) \in K: a=r+\lambda_{1}^{(1)}\right\}$ is the image of a two-sphere $S^{2}(r)$ in $\mathbb{C}^{1,1} / \mathbb{T}^{1} \cong \mathbb{R}^{3}$, and this sphere in turn is the quotient of the three-sphere $S^{3}(2 r)=\left\{|z|^{2}+|w|^{2}=2 r\right\}$ in $\mathbb{C}^{1,1}$. The map $S^{2}(r) \rightarrow \mathbb{D}(r)$ may be thought of as an orthogonal projection to the equatorial plane, whereas the map $S^{3}(2 r) \rightarrow S^{2}(r)$ is the Hopf fibration.

Example 7.2. If $n=1$ and $d=2$ we are considering hypersymplectic quotients of $\mathbb{C}^{2,2}$ by a one-dimensional Abelian group, and the result is a four-dimensional hypersymplectic manifold $M$ with $S^{1}$-symmetry. The map $\beta$ defining $N$ is determined by $u_{1}, u_{2} \in \mathbb{Z}$ not both zero. Without loss of generality we may take $u_{1}>0$. Let us restrict to the non-degenerate case where $u_{2}$ is also non-zero. The moment map $\phi$ on $M$ has image $K=K_{1} \cap K_{2}$, where $K_{k}$ are solid cones in $\mathbb{R} \times \mathbb{C}$ with vertices $V_{k}=\left(\tilde{\lambda}_{k}^{(1)}, \tilde{\lambda}_{k}^{(c)}\right):=\left(\lambda_{k}^{(1)} / u_{k}, \lambda_{k}^{(c)} / u_{k}\right)$. The quotient $M$ is compact if and only if $u_{1}>0>u_{2}$.

Let us successively consider the conditions (F), (S) and (D) of $\$ 4$ for these quotients.

For the freeness condition (F) of Definition 4.1. Proposition 5.6 forces $V_{1} \neq V_{2}$ and imposes the restriction that $u_{k}= \pm 1$ if the vertex $V_{k}$ lies in $K$. Allowable cone configurations include the three given in Figure 7.1, 7.1(a) has $u_{1}>0, u_{2}=1$ and $N=S^{1} ;$ [7.1(b), $u_{1}, u_{2}>0$ and $N=S^{1} \times \mathbb{Z} / \operatorname{gcd}\left(u_{1}, u_{2}\right) ;$ [7.1(c), $u_{1}=1, u_{2}=-1$. Other allowable configurations have $V_{2} \in W_{1} \backslash V_{1}$.

Turning to the smoothness condition (S) of Definition 4.2, assume that the $\mathrm{N}$ action is free. By Remark 5.10, smoothness fails if $V_{2}$ lies in $W_{1}$, since at $V_{2}$ we then have $L=\{1,2\}, J=\{2\}$ and $\mathfrak{n}_{L}=\mathfrak{n}$ has dimension 1 . Thus the configurations of Figure 7.1 are the only candidates for smooth quotients. For Figure 7.1(a), there is no more to check as $\mathfrak{n}_{L}=\{0\}$ at all points of $K$. For Figures 7.1(b) and 7.1](c) we need to consider points $(a, b) \in W_{1} \cap W_{2}$. Here $L=\{1,2\}$ and $J=\varnothing$, so $\mathfrak{n}_{L, J}=\mathfrak{n}_{L}=\mathfrak{n} \cong \mathbb{R}$ and the map $\Lambda_{(a, b)}: \mathfrak{n} \otimes(\mathbb{R} \times \mathbb{C}) \rightarrow \mathbb{C}^{2}$ in (5.5) is

$$
\Lambda_{(a, b)}\left(\left(\begin{array}{l}
x_{1} \\
x_{2}
\end{array}\right) \otimes\left(\theta^{(1)}, \theta^{(c)}\right)\right)=\left(\begin{array}{l}
x_{1}\left(b_{1} \theta^{(1)}+a_{1} \theta^{(c)}\right) \\
x_{2}\left(b_{2} \theta^{(1)}+a_{2} \theta^{(c)}\right)
\end{array}\right),
$$




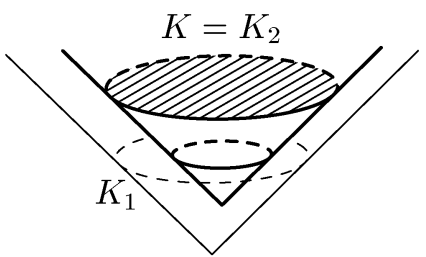

(a) $u_{1} \geqslant u_{2}=1, V_{2} \in \operatorname{Int} K_{1}$

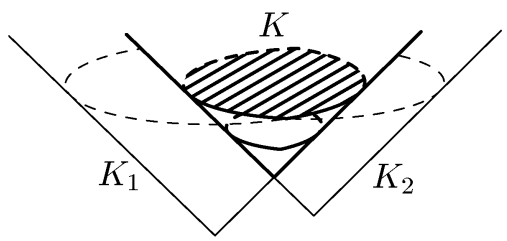

(b) $u_{1}, u_{2}>0, V_{2} \notin K_{1}$

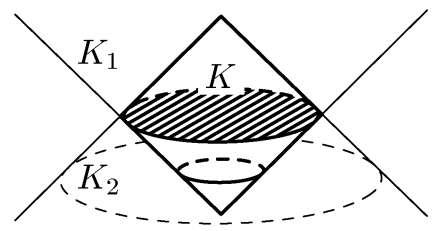

(c) $u_{1}=1=-u_{2}, V_{2} \in \operatorname{Int} K_{1}$

Figure 7.1. Configurations giving smooth quotients from nondegenerate $S^{1}$ - and $S^{1} \times \mathbb{Z} / m$-actions on $\mathbb{C}^{2,2}$.

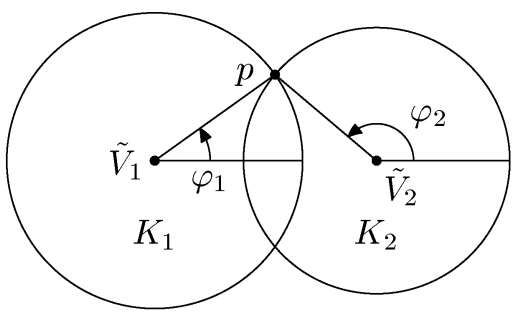

(a) $u_{1}, u_{2}>0$

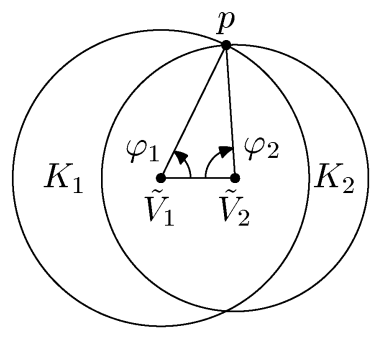

(b) $u_{1}>0>u_{2}$

Figure 7.2. Level sets $a$ constant through $K_{1}$ and $K_{2}$ showing the angles $\varphi_{k}$ for a point $p=(a, b) \in W_{1} \cap W_{2}$. The points $\tilde{V}_{k}$ are the orthogonal projections to this plane of the vertices $V_{k}$.

where $x_{1} u_{1}+x_{2} u_{2}=0$. This is not injective if and only if $b_{1} / a_{1}=-\theta^{(c)} / \theta^{(1)}=$ $b_{2} / a_{2}$ for some $\left(\theta^{(1)}, \theta^{(c)}\right)$. On $W_{k}, a_{k}=\left|b_{k}\right|$, so $b_{k} / a_{k}=e^{\mathbf{i} \varphi_{k}}$. Looking from above, we have Figures 7.2 (a) and 7.2 (b).

One sees that the quotient is smooth when we have $V_{k} \notin W_{j}$ for $k \neq j$. Thus the three configurations of Figure 7.1 give smooth four-dimensional manifolds.

Finally, we turn to the non-degeneracy of the hypersymplectic structure as guaranteed by condition (D) of Definition 4.5, when the quotient is smooth satisfying conditions (F) and (S). Remark 5.12 shows that for (D) to hold we must have $W_{1} \cap W_{2}=\varnothing$. This only occurs when $u_{2}=1$ and $V_{2} \in \operatorname{Int} K_{1}$, as in Figure 7.1(a). Thus the other two configurations give smooth manifolds with a hypersymplectic structure that degenerates along some hypersurface. Indeed in these two cases the fixed point set of the involution $\hat{\sigma}$ of $\$ 6$ is non-empty and Proposition 6.4 applies.

For $u_{2}=1$ and $V_{2} \in \operatorname{Int} K_{1}$ we need to consider Proposition 5.11 in detail. Put $\tilde{a}_{k}=a_{k} / u_{k}=a-\tilde{\lambda}_{k}^{(1)}, \tilde{b}_{k}=b_{k} / u_{k}=b-\tilde{\lambda}_{k}^{(c)}$ and

$$
f_{k}(a, b)=\tilde{a}_{k}^{2}-\left|\tilde{b}_{k}\right|^{2} .
$$


Then $f_{k} \geqslant 0$ on $K_{k}$ with equality on $W_{k}$. Equations (5.6) and (5.7) are now

$$
\begin{gathered}
\zeta_{1}^{2} f_{1}=\frac{1}{4} s^{2}=\zeta_{2}^{2} f_{2}, \\
\zeta_{1} u_{1}+\zeta_{2} u_{2}=0,
\end{gathered}
$$

for some $s \in \mathbb{R}$. In $K=K_{2}$, we have $f_{1}>f_{2}$, since this holds on $W_{2}$ and $f_{1}-f_{2}$ is an increasing linear function of $a$. Fixing $b$, we have $\lim _{a \rightarrow \infty}\left(f_{2} / f_{1}\right)(a, b)=1$, so $f_{2} / f_{1}$ takes all values in $[0,1)$ in $K$. Now (7.1) implies $\zeta_{1}^{2} / \zeta_{2}^{2}=f_{2} / f_{1}$, whereas (7.2) gives $\zeta_{1} / \zeta_{2}=-1 / u_{1}$, since $u_{2}=1$. We conclude that the hypersymplectic structure on this quotient is non-degenerate if and only if $u_{1}=1$.

Topologically, the quotient $M$ from $u_{1}=u_{2}=1$ and $V_{2} \in \operatorname{Int} K_{1}$, is noncompact with two connected components interchanged by the involution $\hat{\sigma}$ of 8 . On each component, $a$ is a Morse function with a single critical point of index 0 . Topologically and smoothly each component of $M$ is $\mathbb{R}^{4}$. The hypersymplectic structure is not in general flat.

To see this, consider the case where $\lambda_{1}^{(c)}=0=\lambda_{2}^{(c)}$, so the quotient carries the Harada-Proudfoot circle action of Remark 5.18, The fixed-point set has, as one component, the image of $w_{1}=0=w_{2}$. This is totally geodesic in $M$ with metric $h$ obtained from the quotient of the set $\left\{\left|z_{1}\right|^{2}-\left|z_{2}\right|^{2}=c_{1}=\lambda_{1}^{(1)}+\lambda_{2}^{(2)}\right\}$ in $\mathbb{C}^{2}$ by the isometric circle action $\left(z_{1}, z_{2}\right) \mapsto\left(e^{\mathbf{i} \theta} z_{1}, e^{-\mathbf{i} \theta} z_{2}\right)$. For the case $c_{1}=1$, writing $z_{1}=\sqrt{1+r^{2}} e^{\mathbf{i} \theta}$ and $z_{2}=r e^{\mathbf{i}(\psi-\theta)}$, we get $h=\frac{2 r^{2}+1}{r^{2}+1} d r^{2}+\frac{r^{2}\left(r^{2}+1\right)}{2 r^{2}+1} d \psi^{2}$, which has curvature $-1 /\left(2 r^{2}+1\right)^{3}$.

7.3. Another circle action. Let us modify the action of \$7.1 so $d=n+1$ and

$$
\begin{gathered}
u_{k}=e_{k}, \quad \text { for } k=1, \ldots, n, \\
u_{n+1}=e_{1}+\cdots+e_{n} .
\end{gathered}
$$

Take $\lambda_{k}^{(i)}=0$ for $i=1,2,3$ and $k=1, \ldots, n$. Put $\lambda_{n+1}^{(1)}=-\lambda<0$ and $\lambda_{n+1}^{(c)}=0$. Then if $a=\left(a_{(1)}, \ldots, a_{(n)}\right) \in \mathbb{R}^{n}$, etc., we have

$$
\begin{gathered}
K_{k}=\left\{(a, b) \in \mathbb{R}^{n} \times \mathbb{C}^{n}: a_{(k)} \geqslant\left|b_{(k)}\right|\right\}, \quad \text { for } k=1, \ldots, n, \\
K_{n+1}=\left\{(a, b) \in \mathbb{R}^{n} \times \mathbb{C}^{n}: \lambda+\sum_{k=1}^{n} a_{(k)} \geqslant\left|\sum_{k=1}^{n} b_{(k)}\right|\right\} .
\end{gathered}
$$

We see immediately that $K$ is the intersection of just $K_{1}, \ldots, K_{n}$ and that $W_{n+1}$ does not meet $K$. As $V_{1} \cap \ldots \cap V_{n}$ lies in $K$ and $\left\{e_{1}, \ldots, e_{n}\right\}$ is a $\mathbb{Z}$-basis for $\mathbb{Z}^{n}$, Proposition 5.6 implies that the action of the circle $N$ is free on $\mu^{-1}(0)$. For smoothness, note that the index sets $L$ and $J$ are both subsets of $\{1, \ldots, n\}$. However, $\mathfrak{n}_{\{1, \ldots, n\}}=\{0\}$ since the restriction of $\beta$ to $\mathfrak{t}_{\{1, \ldots, n\}}=\mathbb{R}^{n} \rightarrow \mathbb{R}^{n}$ is just the identity map. Proposition 5.8 implies that the hypersymplectic quotient $M=\mu^{-1}(0) / N$ is thus smooth. Topologically and smoothly it has two connected components which are copies of $\mathbb{R}^{4 n}$. These components are interchanged by the involution $\hat{\sigma}$.

That the quotient has a non-degenerate hypersymplectic structure may be seen as follows. Using (5.7), we have $\zeta_{1}=\cdots=\zeta_{n}=\zeta$ and $\zeta_{n+1}=-\zeta$. Putting $\tilde{s}=s / 2 \zeta$ the system (5.6) becomes

$$
\begin{gathered}
a_{(k)}^{2}=\left|b_{(k)}\right|^{2}+\tilde{s}_{k}^{2}, \quad \text { for } k=1, \ldots, n, \\
\left(a_{(1)}+\cdots+a_{(n)}+\lambda\right)^{2}=\left|b_{(1)}+\cdots+b_{(n)}\right|^{2}+\left(\tilde{s}_{(1)}+\cdots+\tilde{s}_{(n)}\right)^{2} .
\end{gathered}
$$


Using the triangle inequality, the first $n$-equations imply that the left-hand side of the last equation is strictly greater than the right-hand side for $\lambda>0$. So there is no solution to the degeneracy equations, and we obtain a smooth non-degenerate hypersymplectic structure on two copies of $\mathbb{R}^{4 n}$. The computations of the previous section show that this metric is not flat.

\section{ACKNOWLEDGEMEnTS}

Both authors are members of the EDGE, Research Training Network HPRN-CT2000-00101, supported by The European Human Potential Programme.

\section{REFERENCES}

[AD] A. Andrada and I. Dotti, Double products and hypersymplectic structures on $\mathbb{R}^{4 n}$, Comm. Math. Phys. 262 (2006), no. 1, 1-16. MR2200879

[BD] R. Bielawski and A. Dancer. The geometry and topology of toric hyperkähler manifolds. Comm. Anal. Geom. 8 (2000) 727-759. MR1792372 (2002c:53078)

[De] T. Delzant. Hamiltoniens périodiques et images convexe de l'application moment. Bull. Soc. Math. France 116 (1988) 315-339. MR0984900 (90b:58069)

[FPPW] A. Fino, H. Pedersen, Y. S. Poon and M. Weye Sørensen. Neutral Calabi-Yau structures on Kodaira manifolds. Commun. Math. Phys. 248 (2004), no. 2, 255-268. MR2073135 (2005d:32044)

[Ga] K. Galicki. A generalisation of the momentum mapping construction for quaternionic Kähler manifolds. Commun. Math. Phys. 108 (1987) 117-138. MR0872143 (88f:53088)

[GL] K. Galicki and H. B. Lawson. Quaternionic reduction and quaternionic orbifolds. Math. Annalen 282 (1988) 1-21. MR0960830 (89m:53075)

[Gu] V. Guillemin. Kähler structures on toric varieties. J. Diff. Geom. 40 (1994) 285-309. MR.1293656 (95h:32029)

[HP] M. Harada and N. Proudfoot. Properties of the residual circle action on a hypertoric variety. Pacific J. Math. 214 (2004), no. 2, 263-284. MR2042933(2004k:53139)

[HKLR] N. J. Hitchin, A. Karlhede, U. Lindström and M. Roček. Hyperkähler metrics and supersymmetry. Commun. Math. Phys. 108 (1987) 535-589. MR0877637 (88g:53048)

[H] N. J. Hitchin. Hypersymplectic quotients. Acta Acad. Sci. Tauriensis, supplemento al numero 124 (1990) 169-180.

[Hu] C. M. Hull. Actions for $(2,1)$ sigma models and strings. Nuclear Phys. B 509 (1998), no. 1-2, 252-272. MR 1601779 (98m:81144)

[Ka] H. Kamada. Neutral hyper-Kähler structures on primary Kodaira surfaces. Tsukuba J. Math. 23 (2) (1999) 321-332. MR.1715481 (2000g:53051)

[MS] L. J. Mason and G. A. J. Sparling. Nonlinear Schrödinger and Korteweg-de Vries are reductions of self-dual Yang-Mills. Phys. Lett. A 137 (1989), 29-33. MR0995226 (90d:58169)

Jesus College, Oxford, OX1 3DW, United Kingdom

E-mail address: dancer@maths.ox.ac.uk

Department of Mathematics and Computer Science, University of Southern Denmark, Campusvej 55, DK-5230 Odense M, Denmark

E-mail address: swann@imada.sdu.dk 